\title{
Técnicas de Muestreo sobre una Población a Estudio
}

\author{
Sampling Techniques on a Population Study
}

\author{
Tamara Otzen ${ }^{1,2,3} \&$ Carlos Manterola ${ }^{2,3,4}$
}

OTZEN, T. \& MANTEROLA C. Técnicas de muestreo sobre una población a estudio. Int. J. Morphol., 35(1):227-232, 2017.

RESUMEN: La representatividad de una muestra, permite extrapolar y por ende generalizar los resultados observados en ésta, a la población accesible; y a partir de ésta, a la población blanco. Por ende, una muestra será representativa o no; sólo si fue seleccionada al azar, es decir, que todos los sujetos de la población blanco tuvieron la misma posibilidad de ser seleccionados en esta muestra y por ende ser incluidos en el estudio; y por otro lado, que el número de sujetos seleccionados representen numéricamente a la población que le dio origen respecto de la distribución de la variable en estudio en la población, es decir, la estimación o cálculo del tamaño de la muestra. Es así como el análisis de una muestra permite realizar inferencias, extrapolar o generalizar conclusiones a la población blanco con un alto grado de certeza; de tal modo que una muestra se considera representativa de la población blanco, cuando la distribución y valor de las diversas variables se pueden reproducir con márgenes de error calculables. Entonces, el muestreo tiene por objetivo estudiar las relaciones existentes entre la distribución de una variable en la población blanco y las distribución de ésta variable en la muestra a estudio. Para ello, es fundamental, entre otras cosas definir los criterios de inclusión (características clínicas, demográficas, temporales y geográficas de los sujetos que componen la población en estudio) y de exclusión (características de los sujetos que pueden interferir con la calidad de los datos o la interpretación de los resultados. El objetivo de este manuscrito, es entregar conocimientos generales respecto de las técnicas de muestreo más utilizadas en investigación clínica.

PALABRAS CLAVE: Muestreo; Técnicas de muestreo; Muestreo aleatorio; Muestreo sistemático, Muestreo estratificado; Muestreo por conglomerados; Inferencia.

\section{INTRODUCCIÓN}

La representatividad de una muestra, permite extrapolar y por ende generalizar los resultados observados en ésta, a la población accesible (conjunto de sujetos que pertenecen a la población blanco, que están disponibles para la investigación); y a partir de ésta, a la población blanco. Por ende, una muestra será representativa o no; sólo si fue seleccionada al azar, es decir, que todos los sujetos de la población blanco y accesible, tuvieron la misma posibilidad de ser seleccionados en esta muestra y por ende ser incluidos en el estudio (técnica de muestreo probabilístico); y por otro lado, que el número de sujetos seleccionados representen numéricamente a la población que le dio origen respecto de la distribución de la variable en estudio en la población, es decir, la estimación o cálculo del tamaño de la muestra (Fig. 1). Es así como el análisis de una muestra permite realizar inferencias, extrapolar o generalizar conclusiones a la población blanco con un alto grado de certeza (Dieterich, 1996); de tal modo que una muestra se considera representativa de la población blanco (Fig. 2), cuando la distribu- ción y valor de las diversas variables se pueden reproducir con márgenes de error calculables.

Entonces, el muestreo tiene por objetivo estudiar las relaciones existentes entre la distribución de una variable "y" en una población " $z$ " y las distribución de ésta variable en la muestra a estudio (Hernández Sampieri et al., 2006).

Para ello, es fundamental, entre otras cosas definir los criterios de inclusión (características clínicas, demográficas, temporales y geográficas de los sujetos que componen la población a estudio) y de exclusión (características de los sujetos que pueden interferir con la calidad de los datos o la interpretación de los resultados) (Ávila Baray, 2006; Arias-Gómez et al., 2016). (Fig. 3).

El objetivo de este manuscrito, es entregar conocimientos generales respecto de las técnicas de muestreo más utilizadas en investigación clínica.

\footnotetext{
${ }^{1}$ Universidad de Tarapacá, Arica, Chile.

${ }^{2}$ Centro de Investigaciones Biomédicas, Universidad Autónoma de Chile, Temuco, Chile.

${ }^{3}$ Centro de Excelencia en Estudios Morfológicos y Quirúrgicos (CEMyQ), Universidad de La Frontera, Temuco Chile.

${ }^{4}$ Departamento de Cirugía, Universidad de La Frontera, Temuco, Chile.
} 


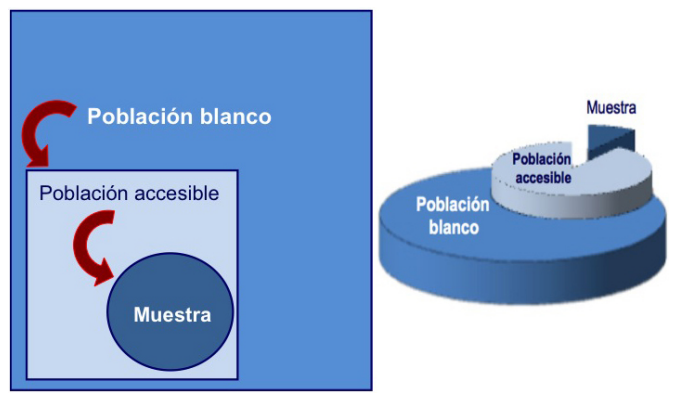

Fig. 1. Una muestra será o no representativa, si fue seleccionada al azar, es decir todos los sujetos de la población blanco tienen la misma posibilidad de ser seleccionados en la muestra. La población accesible es el conjunto de sujetos que pertenecen a la población blanco, que están disponibles para la investigación.

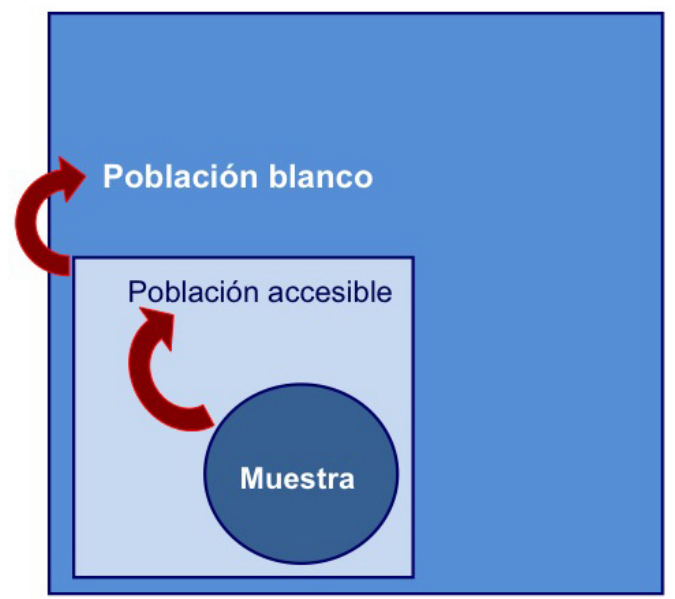

Fig. 2. Generalización de resultados. La representatividad de la muestra, permite extrapolar los resultados a la población de accesible y de ésta a la población general.

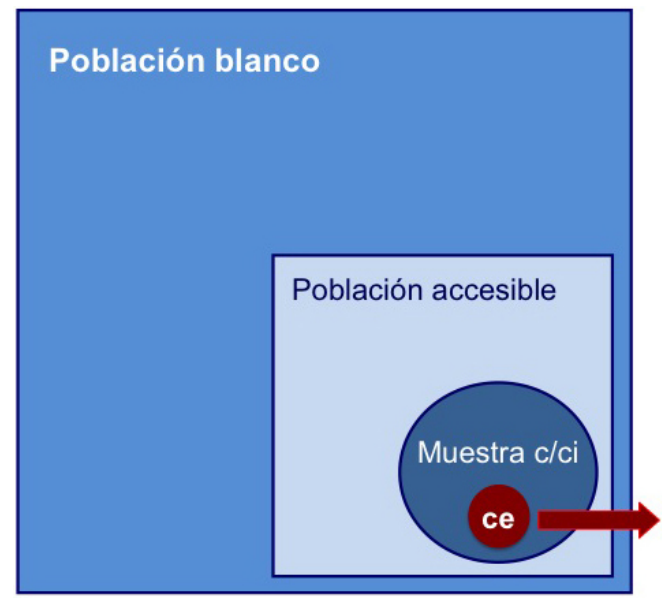

Fig. 3. Criterios de elegibilidad. Los criterios de inclusión corresponden a aquellas características clínicas, demográficas, temporales y geográficas de los sujetos que componen la población en estudio. Por su parte, los criterios de exclusión corresponden a las características de los sujetos que pueden interferir con la calidad de los datos o la interpretación de los resultados.

\section{Técnicas de Muestreo}

Una muestra puede ser obtenida de dos tipos: probabilística y no probabilística. Las técnicas de muestreo probabilísticas, permiten conocer la probabilidad que cada individuo a estudio tiene de ser incluido en la muestra a través de una selección al azar. En cambio, en las técnicas de muestreo de tipo no probabilísticas, la selección de los sujetos a estudio dependerá de ciertas características, criterios, etc. que él (los) investigador (es) considere (n) en ese momento; por lo que pueden ser poco válidos y confiables o reproducibles; debido a que este tipo de muestras no se ajustan a un fundamento probabilístico, es decir, no dan certeza que cada sujeto a estudio represente a la población blanco (Walpole \& Myers, 1996; Ávila Baray; Arias-Gómez et al.).

\section{Técnicas de muestreo probabilístico.}

a) Aleatorio simple: Garantiza que todos los individuos que componen la población blanco tienen la misma oportunidad de ser incluidos en la muestra. Esta significa que la probabilidad de selección de un sujeto a estudio " $x$ " es independiente de la probabilidad que tienen el resto de los sujetos que integran forman parte de la población blanco. Por ejemplo: ante la siguiente pregunta de investigación ¿Cuál es la muestra necesaria para establecer la prevalencia de cambios inflamatorios en biopsias hepáticas de pacientes con colangitis aguda (CA)? Un muestreo aleatorio simple aplicaría de la siguiente forma: entre todos los sujetos con CA, seleccionar al azar un subgrupo que los represente (Fig. 4).

b) Aleatorio estratificado: Se determina los estratos que conforman la población blanco para seleccionar y extraer de ellos la muestra (se define como estrato a los subgrupos de unidades de análisis que difieren en las características que van a ser analizadas). La base de la estratificación se basa en variable como edad, sexo, nivel socioeconómico, etc. Entonces, se divide la población compuesta por " $\mathrm{N}$ " individuos, en " $\mathrm{x}$ " subpoblaciones o estratos, con base a variables importantes para la conducción del estudio, y de tamaños respectivos N1, N2, N3, N4 ..., Nk; y realizando en cada una de estos estratos, muestreos aleatorios simples de tamaño ni; para finalmente definir cuantos elementos de la muestra se han de seleccionar de cada uno de los estratos; para lo cual se dispone de las siguientes opciones: asignación proporcional (el tamaño de la muestra de cada estrato es proporcional al tamaño del estrato que le dio origen, respecto a la población total) y asignación óptima (el tamaño de la muestra de cada estrato, son definidos por quien hace el muestreo) (Bai et al., 2013). Por ejemplo: ante la pregunta ¿Cuál es la muestra necesaria para establecer la prevalencia de cambios inflamatorios en biopsias hepáticas de pacientes con CA? Un muestreo aleatorio estratificado aplicaría de la siguiente forma: entre todos los sujetos con CA, agrupar en forma aleatoria por características de interés como: gravedad de la enfermedad (leve, moderado, grave); intensidad de la fiebre (febril, afebril, hipotérmico); leucocitosis (con y sin leucocitosis); nivel de bilirrubina total (hasta 2,0; 2,1 a 4,0; 4,1 a 6,9; 7,0 o más), etc. (Fig. 5). 


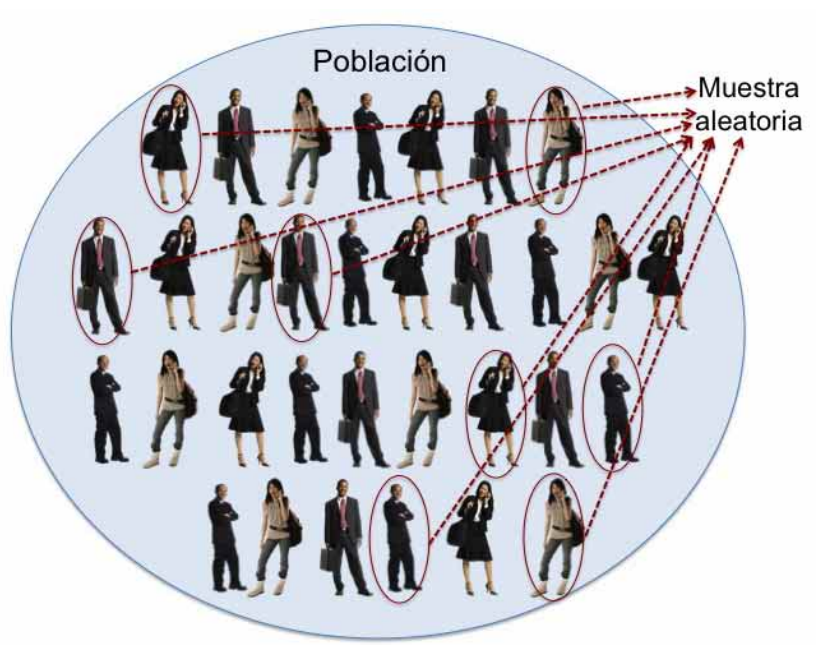

Fig. 4. Muestreo aleatorio simple. A partir de la población blanco, se seleccionan al azar el número de sujetos necesario para completar la muestra necesaria a estudiar.

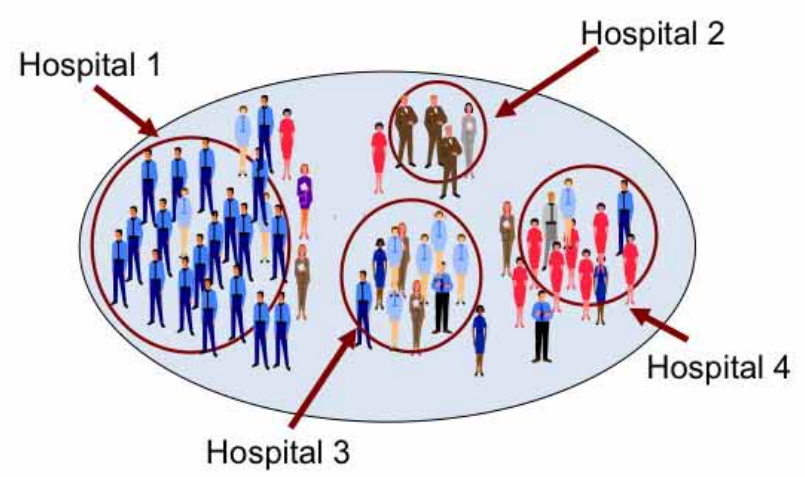

Fig. 5. Muestreo aleatorio estratificado. Se seleccionan al azar una muestra de los 4 centros hospitalarios (estratos), que componen la población total de una región del país (población blanco); y de cada estrato, se selecciona un número de sujetos que lo representan.

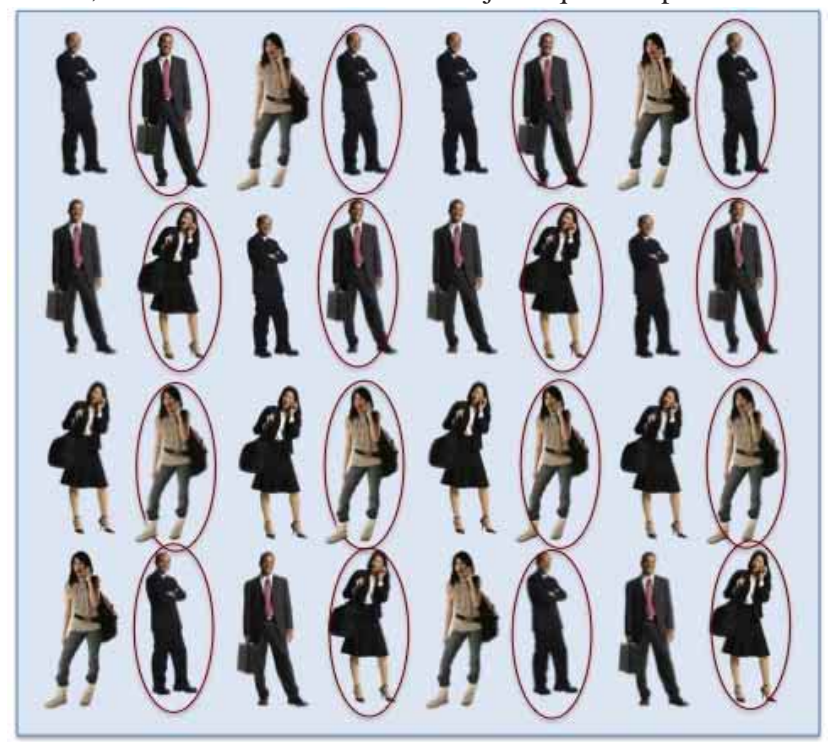

Fig. 6. Muestreo aleatorio sistemático. Se selecciona de forma sistemática cada hésimo caso de la población blanco. c) Aleatorio sistemático: Cuando el criterio de distribución de los sujetos a estudio en una serie es tal, que los más similares tienden a estar más cercanos. Este tipo de muestreo suele ser más preciso que el aleatorio simple, debido a que recorre la población de forma más uniforme. De esta modo, se seleccionará cada hésimo caso (Arias-Gómez et al.). Por ejemplo: ante la pregunta ¿Cuál es la muestra necesaria para establecer la prevalencia de cambios inflamatorios en biopsias hepáticas de pacientes con CA? Un muestreo aleatorio sistemático aplicaría de la siguiente forma: entre todos los sujetos con CA, seleccionar aquellos que ingresan los días impares del mes, o aquellos cuya primer dígito del RUT sea par, hasta completar la muestra estimada (Fig. 6).

d) Por conglomerados: Consiste en elegir de forma aleatoria ciertos barrios o conglomerados dentro de una región, ciudad, comuna, etc., para luego elegir unidades más pequeñas como cuadras, calles, etc. y finalmente otras más pequeñas, como escuelas, consultorios, hogares (una vez elegido esta unidad, se aplica el instrumento de medición a todos sus integrantes). Si se desea realizar un estudio de prevalencia o una encuesta en habitantes de una localidad, el muestreo aleatorio simple es complejo y de alto costo, ya que estudiar una muestra de tamaño "n", supone enviar encuestadores a " $x$ " puntos diferentes de la misma; de tal forma que en cada uno de estos puntos, sólo se aplicará una encuesta (Hund et al., 2015). Por ello, es que en este tipo de casos se sugiere aplicar muestreo por conglomerados, pues son más económicos y eficientes. En este tipo de muestreo, los sujetos a estudio, se encuentran incluidos en lugares físicos o geográficos (conglomerados); por ende, resulta imprescindible diferenciar entre sujetos a estudio (quiénes va a ser medidos) y unidad muestral (conglomerado a través del cual se logra acceder a los sujetos a estudio) (Fig. 7).

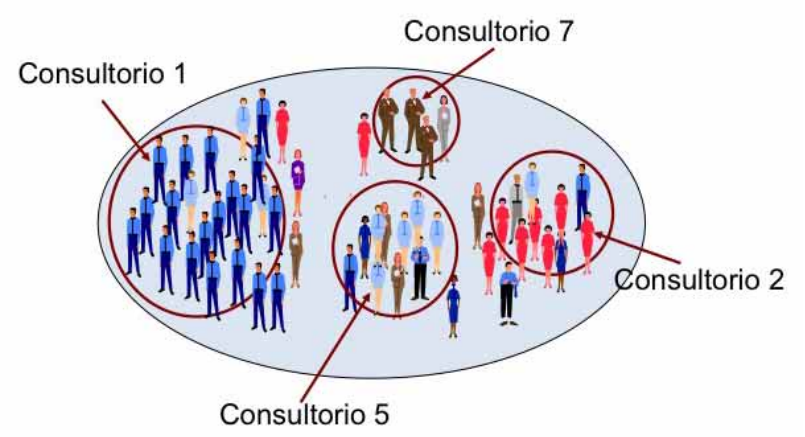

Fig. 7. Muestreo por conglomerados. Se seleccionan al azar una muestra de 4 consultorios de salud (conglomerados), de los 9 existentes en una comuna; y de cada conglomerado, se mide la variable de interés a todos los sujetos que deben consultar en ellos. 
Por ejemplo: ante la pregunta ¿Cuál es la muestra necesaria para establecer la prevalencia de cambios inflamatorios en biopsias hepáticas de pacientes con CA? Un muestreo aleatorio por conglomerados aplicaría de la siguiente forma: entre todos los sujetos con CA de la Región de la Araucanía, seleccionar en forma aleatoria aquellos provenientes de los hospitales de Angol, Imperial y de la Clínica Alemana de Temuco (conglomerados que representarán a todos los centros sanitarios de esta región).

\section{Técnicas de muestreo no probabilístico}

a) Intencional: Permite seleccionar casos característicos de una población limitando la muestra sólo a estos casos. Se utiliza en escenarios en las que la población es muy variable y consiguientemente la muestra es muy pequeña. Por ejemplo, entre todos los sujetos con CA, seleccionar a aquellos que más convengan al equipo investigador, para conducir la investigación.

b) Por conveniencia: Permite seleccionar aquellos casos accesibles que acepten ser incluidos. Esto, fundamentado en la conveniente accesibilidad y proximidad de los sujetos para el investigador. Por ejemplo, entre todos los sujetos con CA, solamente aquellos que se encuentren hospitalizados en el Hospital Regional de Temuco.

c) Accidental o consecutivo: Se fundamenta en reclutar casos hasta que se completa el número de sujetos necesario para completar el tamaño de muestra deseado. Estos, se eligen de manera casual, de tal modo que quienes realizan el estudio eligen un lugar, a partir del cual reclutan los sujetos a estudio de la población que accidentalmente se encuentren a su disposición. Es similar al muestreo por conveniencia, excepto que intenta incluir a todos los sujetos accesibles como parte de la muestra. Por ejemplo, entre todos los sujetos con CA, seleccionar los primeros 50 incluibles que lleguen al servicio de urgencias del Hospital Regional de Temuco.

\section{EJEMPLOS}

Ejemplo 1: En un estudio cuyo objetivo fue determinar la eficiencia de los programas de tamizaje de cáncer gástrico por endoscopia digestiva alta en 4 centros de atención primaria en de la ciudad de Niigata, cuya población era de 811.000 habitantes; para optimizar la productividad de estos programas, se realizó un muestreo no probabilístico por conveniencia de 44 sujetos asintomáticos mayores de 40 años, que fueron estudiados de forma gratuita. En ellos se midieron los tiempos, número de personas y costes involucradas en el procedimiento. Se verificó que los tiempos de preparación y premedicación; del procedimiento propiamente tal y de los cuidados post-procedimiento (recuperación y limpieza); representaron el 34,1\%; 10,6 \% y $54,4 \%$ del tiempo total de la endoscopia, respectivamente. Concluyendo de esta manera que la mayor parte del tiempo necesario para completar la detección del cáncer gástrico vía endoscópica es consumido por la preparación, la premedicación y los procedimientos posteriores, en los que las enfermeras desempeñan un papel clave (Goto $e t$ al., 2014).

Ejemplo 2: En un estudio conducido en Irán, cuyo objetivo fue evaluar la epidemiología de la ERGE y sus factores de riesgo de en una población basada en el estudio de Mashhad (segunda ciudad más grande de Irán); se realizó un estudio de corte transversal, aplicando el cuestionario generado por la Clínica Mayo a un total de 1685 sujetos; para lo cual se desarrolló una técnica de muestreo por conglomerados en 50 locaciones seleccionadas a partir de los códigos de área eléctrica, incluyendo 50 participantes por cada locación. Encontraron un 25,7 \% de síntomas de ERGE en la población estudiada; y los siguientes factores de riesgo: tabaquismo, consumo de anti inflamatorios no esteroidales, enfermedades crónicas, consumo de te y café, sobrealimentación y ERGE en la pareja (Vossoughinia et al., 2014).

\section{Ventajas y Desventajas de la Aplicación de Técnicas de Muestreo}

Se puede comentar que cada técnica de muestreo tiene sus ventajas y desventajas (Tabla I); sin embargo, en términos generales existen ventajas y desventajas comunes para todas ellas:

\section{VENTAJAS}

Reducción de costos: Los costes de un estudio serán menores si los datos de interés se pueden obtener a partir de una muestra de la población blanco. Por ejemplo, cuando se realizan estudios de prevalencia de un evento de interés, es más económico medir una muestra representativa de 1500 sujetos de la población blanco, que a los 250.000 individuos que la componen.

Eficiencia: Al trabajar con un número reducido de sujetos a estudio, representativos de la población blanco; el tiempo necesario para conducir el estudio y obtener resultados y conclusiones será ostensiblemente menor.

\section{DESVENTAJA}

Inadecuada representación de la población blanco: Esto puede ocurrir si se decide trabajar con muestras pequeñas. 
Tabla I. Ventajas y desventajas de los distintos tipos de muestreo probabilístico.

\begin{tabular}{|c|c|c|c|}
\hline Tipo & Características & Ventajas & Desventajas \\
\hline A leatorio simple & $\begin{array}{l}\text { - Selección de una muestra de } \\
\text { tamaño "n", a partir de una } \\
\text { población de "N" unidades. } \\
\text { - Cada elemento tiene la } \\
\text { misma probabilidad de } \\
\text { inclusión }(\mathrm{n} / \mathrm{N}) \text {. }\end{array}$ & $\begin{array}{l}\text { - Sencillo y de fácil } \\
\text { comprensión. } \\
\text { - Cálculo rápido de medias } \\
\text { y varianzas. } \\
\text { - Existen paquetes } \\
\text { informáticos para analizar } \\
\text { los datos. }\end{array}$ & $\begin{array}{l}\text { - Requiere listado completo } \\
\text { de toda la población. } \\
\text { - Al trabajar con muestras } \\
\text { pequeñas puede no } \\
\text { representar de forma } \\
\text { adecuada a la población. }\end{array}$ \\
\hline $\begin{array}{l}\text { A leatorio } \\
\text { sistemático }\end{array}$ & $\begin{array}{l}\text { - C onseguir un listado de los } \\
\text { "N" elementos de la } \\
\text { población } \\
\text { - D eterminar tamaño de } \\
\text { muestra " } n \text { ". } \\
\text { - D efinir intervalo k= N/n. } \\
\text { - E legir número aleatorio, } \\
\text { entre } 1 \text { y k. } \\
\text { - S elección de los elementos } \\
\text { de la lista. }\end{array}$ & $\begin{array}{l}\text { - Fácil de aplicar. } \\
\text { - No siempre es necesario } \\
\text { tener listado de la } \\
\text { población. } \\
\text { - Cuando la población está } \\
\text { ordenada, asegura } \\
\text { cobertura de unidades de } \\
\text { todos los tipos. }\end{array}$ & $\begin{array}{l}\text { - Si la constante de muestreo } \\
\text { está asociada con el } \\
\text { evento de interés, las } \\
\text { estimaciones obtenidas a } \\
\text { partir de la muestra } \\
\text { pueden contener sesgo de } \\
\text { selección. }\end{array}$ \\
\hline $\begin{array}{l}\text { A leatorio } \\
\text { estratificado }\end{array}$ & $\begin{array}{l}\text { - En ciertas ocasiones } \\
\text { resultará conveniente } \\
\text { estratificar la muestra según } \\
\text { variables de interés. } \\
\text { - Para ello se ha de conocer la } \\
\text { composición estratificada de } \\
\text { la población objeto. } \\
\text { - Una vez calculado el tamaño } \\
\text { de la muestra, este se reparte } \\
\text { entre los distintos estratos de } \\
\text { la población usando regla de } \\
\text { tres. }\end{array}$ & $\begin{array}{l}\text { - Tiende a asegurar que la } \\
\text { muestra represente } \\
\text { adecuadamente a la } \\
\text { población en función de } \\
\text { variables seleccionadas. } \\
\text { - Se obtienen estimaciones } \\
\text { más precisas. } \\
\text { - Su objetivo es conseguir } \\
\text { una muestra lo más } \\
\text { semejante a la población } \\
\text { en lo que a la o las } \\
\text { variables estratificadoras } \\
\text { se refiere. }\end{array}$ & $\begin{array}{l}\text { - Se ha de conocer la } \\
\text { distribución en la } \\
\text { población de las variables } \\
\text { utilizadas para la } \\
\text { estratificación. }\end{array}$ \\
\hline $\begin{array}{l}\text { A leatorio por } \\
\text { conglomerados }\end{array}$ & $\begin{array}{l}\text { - Se realizan varias fases de } \\
\text { muestreo sucesivas. } \\
\text { - La necesidad de listados de } \\
\text { unidades se limita a unidades } \\
\text { de muestreo seleccionadas } \\
\text { en la etapa anterior. }\end{array}$ & $\begin{array}{l}\text { Eficiente en poblaciones } \\
\text { grandes y dispersas. } \\
\text { - Es preciso tener un } \\
\text { listado de las unidades } \\
\text { primarias de muestreo. }\end{array}$ & $\begin{array}{l}\text { - El error estándar es mayor } \\
\text { que en otros tipos de } \\
\text { muestreo. } \\
\text { - El cálculo del error } \\
\text { estándar es complejo. }\end{array}$ \\
\hline
\end{tabular}

Por ejemplo, es muy común ver publicaciones en las que se comparan 50 sujetos tratados con la intervención en estudio versus 50 con la intervención estándar. Este es un número muy recurrente, pero posiblemente conlleva una mala representación de la población a la que se desea inferir los resultados.
OTZEN, T. \& MANTEROLA C. Sampling techniques on a population study. Int. J. Morphol., 35(1):227-232, 2017.

SUMMARY: The representativeness of a sample allows extrapolating and therefore generalizes the results observed in this, the accessible population, and from this, to the target population. 
Thus, a sample will be representative or not, only if it was selected at random, i.e., that all the subjects of the target population had the same possibility of being selected in this sample and therefore be included in the study, and on the other hand, that the number of subjects selected numerically represent the population that gave rise to it with respect to the distribution of the variable under study in the population, that is, the estimation of the sample size. Consequently, the analysis of a sample allows us to make inferences or generalize conclusions to the target population with a high degree of certainty, such that a sample is considered representative of the target population, when the distribution and value of the different variables can be reproduced with calculable error margins. So, the aim of sampling is to study the relationships between the distribution of a variable in the target population and the distribution of the same variable in the study sample. For this purpose, it is essential, among other things, to define the inclusion criteria (clinical, demographic, temporal and geographical characteristics of the subjects that make up the study population) and the exclusion criteria (characteristics of the subjects that may interfere with the quality of the data Or the interpretation of results). The aim of this manuscript is to provide general knowledge regarding sampling techniques most commonly used in clinical research.

KEY WORDS: Sampling Studies; Study; Sampling; Probability Sample; Samples; Probability.

\section{REFERENCIAS}

Ávila Baray, H. L. Introducción a la Metodología de la Investigación. Edición electrónica. Cuauhtémoc (Chihuahua), Instituto Tecnológico de Cd. Cuauhtémoc, 2006 Disponible en: http://www.eumed.net/libros-gratis/2006c/203/index.htm

Arias-Gómez, J.; Villasís-Keever, M. Á. \& Miranda-Novales, M. G. The research protocol III. Study population. Rev. Alerg. Mex., 63(2):201-6, 2016.

Bai, X.; Tsiatis, A. A. \& O'Brien, S. M. Doubly-robust estimators of treatment-specific survival distributions in observational studies with stratified sampling. Biometrics, 69(4):830-9, 2013.

Dieterich, H. Nueva Guía para la Investigación Científica. Ciudad de México, Editorial Planeta Mexicana, 1996. Disponible en: http:// www.ceuarkos.com/heinz.pdf

Goto, R.; Arai, K.; Kitada, H.; Ogoshi, K. \& Hamashima, C. Labor resource use for endoscopic gastric cancer screening in Japanese primary care settings: A work sampling study. PLoS One, 9(2):e88113, 2014.

Hernández Sampieri, R.; Fernández-Collado, C. \& Baptista Lucio, P. Metodología de la Investigación. $4^{\mathrm{a}}$ ed. Ciudad de México, McGraw-Hill, 2006. Disponible en: https://competenciashg.files.wordpress.com/2012/ 10/sampieri-et-al-metodologia-de-la-investigacion-4ta-edicionsampieri-2006_ocr.pdf

Hund, L.; Bedrick, E. J. \& Pagano, M. Choosing a cluster sampling design for lot quality assurance sampling surveys. PLoS One, 10(6):e0129564, 2015.

Vossoughinia, H.; Salari, M.; Mokhtari Amirmajdi, E.; Saadatnia, H.; Abedini, S.; Shariati, A.; Shariati, M. \& Khosravi Khorashad, A. An epidemiological study of gastroesophageal reflux disease and related risk factors in urban population of mashhad, Iran. Iran. Red Crescent Med. J., 16(12):e15832, 2014.

Walpole, R. E. \& Myers, R. H. Probabilidad y Estadística. 4a . ed. Ciudad de México, McGraw-Hill, 1996.
Dirección para correspondencia:

Dr. Carlos Manterola, MD, PhD.

Departamento de Cirugía y CEMyQ

Universidad de La Frontera

Temuco

CHILE

Teléfono: $56-45-2325760$

Fax: 56-45-2325761

E-mail: carlos.manterola@ufrontera.cl

Received: 15-09-2016

Accepted: 19-12-2016 